\title{
Admixture and ethno-specific alleles: missing links for global pharmacogenomics
}

\author{
"Admixture is deemed a potential confounder that may \\ give rise to spurious associations in genetic studies due to \\ varying degree of parental backgrounds among individuals \\ in the population."
}

First draft submitted: 30 June 2016; Accepted for publication: 8 July 2016; Published online: 18 August 2016

Keywords: admixture $\bullet$ Caribbean Hispanics • warfarin pharmacogenomics

We and colleagues from the University of Puerto Rico Medical Sciences Campus, the VA Caribbean Healthcare System and the Hartford Hospital-affiliated Brownstone outpatient clinic, CT, USA, have recently published in PLOS ONE a research article [1] on the use of admixture measures to further improve predictability of a pharmacogenetic-guided algorithm for effective warfarin dosing refinements in Caribbean Hispanic patients as well as to adjust for population stratification within this Latino subgroup. A clinical protocol was conducted as an open-label, population-based, observational, retrospective cohort study (ClinicalTrial.gov Identifier NCT01318057) in a derivation (learning) sample of 255 eligible patients (mostly elderly and males) of the Commonwealth of Puerto Rico, and later externally validated in another cohort of 55 individuals from Hartford, CT, USA (both males and females, age range: 23-90 years old). The purpose of this study protocol was to develop and test the clinical validity of a novel admixture-adjusted pharmacogenetic model to individually predict and refine optimal warfarin dosing in Caribbean Hispanic patients. If proven to be superior to the current standard of care, this new method to estimate ideal warfarin doses will help physicians achieve the desired medical endpoints on admixed Caribbean Hispanic patients sooner and stabilize them longer on target with lesser risks of adverse events (e.g., serious bleedings, strokes, among others).
To derive the final pharmacogenetic model, a multiple linear regression analysis of effective warfarin doses versus relevant genotypes, admixture, clinical and demographic factors was performed. To compute individual ancestry proportions (a.k.a., admixture indexes), a Bayesian's clustering of individual genotypes from 332 physiogenomic ancestry markers [2] was performed, according to the maximal allele sharing, using STRUCTURE v2.3.4 software package [3]. The parameters applied were ten independent runs with 70,000 burn-in steps and 30,000 Markov chain Monte Carlo replicates assuming three ancestral populations $(\mathrm{K}=3)$ in admixture model, allele frequencies correlated and the parameter USEPOPINFO = 1. A trihybrid model was chosen to reflect admixture from Native Americans (Tainos Arawaks), west Africans and European ancestors as well as to match previous reports [4] based on current knowledge of migration history in the Caribbean basin. For the purpose of the regression analysis, this admixture index was treated as a categorical variable as follows: 1 = Europeans; 2 = Native Americans; 3 = African ancestry; 4 = Admixed (i.e., those individuals not clearly positioned toward any cluster or vertex of the STRUCTURE triangle plot but rather located near the middle of the triangle).

An admixture-adjusted pharmacogenetic model was developed with 13 significant 'explanatory' variables that explained
Jorge Duconge

Author for correspondence:

School of Pharmacy, University of Puerto Rico Medical Sciences Campus, PO Box 365067, San Juan, PR 00936, USA

Jorge.duconge@upr.edu

\section{Gualberto Ruaño}

Pharmaceutical Sciences Department, Genomas Inc., Hartford, CT 06102, USA 
more than two-thirds of the observed variance in the stable warfarin dose in Caribbean Hispanics [1]. Among the predictors of warfarin dose variability in the final model, the effect on dose of all CYP2C9 polymorphisms combined was $-18 \%$; whereas, the effect of both VKORC1-AA and VKORC1-GA status combined was $-13 \%$. CYP4F2*3 18000G $>\mathrm{A}$ (V433M, rs2108622) and $\mathrm{NQOI}^{*} 2$ c.559C $>\mathrm{T}$ (P187S, rs1800566) variants were independently associated with a 17 and $10 \%$ increase of the dose per variant allele, respectively; whereas, the admixture index decreases the dose by $7 \%$. Notably, the effect of admixture on dose reduction increases from 7 to $11.48 \%$ when this variable is tested in the absence of the above genotypes, suggesting admixture index is partially capturing the missing heritability if genotypes are canceled out of the model. The Africanrelated rare 'loss-of-function' variant $\mathrm{CYP}^{2} \mathrm{C}^{*} 8$ (c.449G>A; p.R150H) and two other polymorphisms in the promoter region of the CYP2C9 gene locus (i.e., c.-1188T>C and c. $-1766 \mathrm{~T}>\mathrm{C}$, in strong linkage disequilibrium with $C Y P 2 C 9^{*} 8$ ), were detected in $1.2 \%$ of patients (all heterozygous) and became part of the model equation to explain $31 \%$ decrease of the dose. The ${ }^{*} 8$ allele was originally found in AfricanAmericans [5-7] and has been previously associated with 30\% decrease of CYP2C9 enzyme activity for $S$-warfarin conversion [8].

“

"...both individuals' ethno-specific genotypes and unique admixture metrics increase predictability of pharmacogenetic refinement models when applying this approach to admixed Caribbean Hispanics."

Our findings supported the postulated hypothesis that both individual's ethno-specific genotypes and unique admixture metrics increase predictability of pharmacogenetic refinement models when applying this approach to admixed Caribbean Hispanics. Indeed, results suggest that stable warfarin doses are better predicted in Caribbean Hispanics by using our novel admixture-adjusted, genotype-guided refinement algorithm than if a clinical model that excluded genotypes and admixture is used (i.e., $\mathrm{R}^{2}=$ 0.70 vs $0.60 ; \mathrm{MAE}=0.72$ vs $0.99 \mathrm{mg} / \mathrm{day}$, respectively; $\mathrm{p}<0.001)$. MAE stands for mean absolute error, defined as the mean of the absolute values for the difference between the model predicted and actual doses. Likewise, our model also outperformed two early published pharmacogenetic algorithms (i.e., IWPC pharmacogenetic initiation algorithm [9] and the Lenzini et al. pharmacogenetic refinement model [10]) in predicting effective dose in this population $\left(\mathrm{R}^{2}=0.70\right.$ vs 0.34 and $0.27 ; \mathrm{MAE}=0.72$ vs 1.3 and $1.2 \mathrm{mg} /$ days, respectively). Moreover, external validation in 55 Caribbean Hispanic patients from an independent cohort accounted for $58 \%$ of dose variability $(\mathrm{MAE}=0.89 \mathrm{mg} /$ day, $24 \%$ mean bias $)$. Notably, $\sim 50 \%$ of dose predictions in patients at the highest risk of adverse events resulted in ideal doses (i.e., MAE value was $<1 \mathrm{mg}$ /day, falling within $20 \%$ of the actual dose that is a well-accepted criterion for accuracy in dose estimation) when using the admixture-driven pharmacogenetic algorithm as compared with only $29 \%$ when using the clinical nongenetic algorithm $(\mathrm{p}<0.001)$.

On the other hand, a richer genetic diversity in the highly admixed population of Caribbean Hispanics, as compared with other parental groups (e.g., Caucasians), was confirmed by the observed frequency distribution of multiple ethno-specific polymorphic variants across several warfarin-related pharmacogenes. Some of these polymorphisms had been identified before exclusively in either people of African ancestry (e.g., ${ }^{*} 5,{ }^{*} 6,{ }^{*} 8,{ }^{*} 11$ ) or Caucasians (e.g., ${ }^{*} 2,{ }^{*} 3$ ), but not coexisting in a single population (i.e., leave alone a given individual). Besides, the combination of $\mathrm{CYP}^{2} \mathrm{~F}^{*} 3$ and $\mathrm{NQOI}^{*} 2$ 'resistant' variants, identified in as many as 20.4 and $17.7 \%$ of individuals from the study cohort, had only been previously described to be associated with higher warfarin dose requirements in another US Hispanic population [11].

Multiple linear regression analysis routinely used to derive pharmacogenetic models is data driven and hence population dependent. Most of the existing pharmacogenetic algorithms were developed in cohorts of patient of mostly European descent, and therefore they often include only the $C Y P 2 C 9^{*} 2,{ }^{*} 3$ allele variants and the VKORC1-1639G $>\mathrm{A}$ haplotype status commonly found among Caucasians. Uncommon mutations in Europeans are generally overlooked and, consequently, the utility of existing algorithms is limited in patients with mixed ancestry like Caribbean Hispanics. Consequently, it is not surprising a lack of fully endorsement by professional organizations and payers. The COAG trial (NCT00839657) raised concerns about expected clinical benefits of genotyping patients to improve warfarin dose predictions; however, we strongly believe that failure to account for ethno-specific genetic polymorphisms relevant in underrepresented admixed populations, as well as adjustments by admixture, lead to significant dosing error.

Despite the fact that a few prior pharmacogenetic algorithms have certainly added ethnicity or 'selfreported' race as a proxy for ancestry, this is to our knowledge the first time a pharmacogenetic study includes a measure of admixture in warfarin dose pre- 
dictions. Admixture is deemed a potential confounder that may give rise to spurious associations in genetic studies due to varying degree of parental backgrounds among individuals in the population [12-15]. We took the alternative view that admixture can be a quantitative predictor of response when appropriately indexed. In our work, we found a significant association between the proportion of individual Amerindian ancestry and low warfarin dose requirements. We speculate that individuals with significant Amerindian ancestry on their genetic make-up, particularly at the VKORC1 locus, are more likely to need lower doses because of a relatively higher odd to carry the VKORC1 haplotype A. Admixed individuals are unique in terms of frequency distribution, combination of allelic variants across their genomes and linkage disequilibrium by ancestry [16-18]. Such differences might have significant consequences for associations between genotypes and warfarin dosing.

\section{Conclusion}

Taking altogether, we firmly believe admixture indexes and certain ethno-specific alleles are both clinically relevant predictors for algorithmically computed warfarin doses in Caribbean Hispanics given the welldocumented stratification of this population, marked genomic diversity and ethno-geographic heterogeneity and distinctive clines of admixture proportions across the region. Future studies are warranted to confirm clinical utility of this new approach in the admixed population of Caribbean Hispanics.

\section{References}

1 Duconge J, Ramos AS, Claudio K et al. A novel admixturebased pharmacogenetic approach to refine warfarin dosing in Caribbean Hispanics. PLoS ONE 11(1), e0145480 (2016).

2 Ruaño G, Duconge J, Windemuth A et al. Physiogenomic analysis of the Puerto Rican population. Pharmacogenomics 10(4), 565-577 (2009).

3 Pritchard JK, Stephens M, Donnelly P. Inference of population structure using multilocus genotype data. Genetics 155, 945-959 (2000).

4 Via M, Gignoux C, Roth LA et al. History shaped the geographic distribution of genomic admixture on the island of Puerto Rico. PLoS ONE 6, e16513 (2011).

5 Cavallari LH, Langaee TY, Momary KM et al. Genetic and clinical predictors of warfarin dose requirements in African Americans. Clin. Pharmacol. Ther. 87, 459-464 (2010).

6 Drozda K, Wong S, Patel SR et al. Poor warfarin dose prediction with pharmacogenetic algorithms that exclude genotypes important for African Americans. Pharmacogenet. Genomics 25(2), 73-81 (2015).

\section{Acknowledgements}

We also thank Genomas Inc., Hartford, CT, and the UPR-MSC RCMI Center for Genomics in Health Disparities and Rare Disorders for their support and for providing the resources and facilities for performing genetic assays.

\section{Disclaimer}

The contents of this publication are solely the responsibility of the authors and do not represent the official views of the VA Caribbean Healthcare System, the Department of Veterans Affairs, the NIH or the United States Government.

\section{Financial \& competing interests disclosure}

$G$ Ruaño is founder and President of Genomas Inc. J Duconge also holds a without compensation (WOC) employment status with the Pharmacy Service, VA Caribbean Healthcare Systems (VACHS), in San Juan, Puerto Rico. The material presented herein is the result of work supported in part by the grant number SC1 HL123911 from the National Heart, Lung, and Blood Institute (NHLBI), with resources from and the use of facilities at the Veteran Affairs Caribbean Health System (VACHS) in San Juan, Puerto Rico. Genomas, Inc. provided support in the form of salaries for $G$ Ruaño, but did not have any additional role in the study design, data collection and analysis, decision to publish or preparation of this letter. The authors have no other relevant affiliations or financial involvement with any organization or entity with a financial interest in or financial conflict with the subject matter or materials discussed in the manuscript apart from those disclosed.

No writing assistance was utilized in the production of this manuscript.

7 Scott SA, Jaremko M, Lubitz SA, Kornreich R, Halperin JL, Desnick RJ. CYP2C9*8 is prevalent among AfricanAmericans: implications for pharmacogenetic dosing. Pharmacogenomics 10(8), 1243-1255 (2009).

8 Liu $\mathrm{Y}$, Jeong $\mathrm{H}$, Takahashi $\mathrm{H}$ et al. Decreased warfarin clearance associated with $C Y P 2 C 9 \mathrm{R} 150 \mathrm{H}\left({ }^{*} 8\right)$ polymorphism. Clin. Pharmacol. Ther. 91(4), 660-665 (2012).

9 International Warfarin Pharmacogenetics Consortium, Klein TE, Altman RB et al. Estimation of the warfarin dose with clinical and pharmacogenetic data. N. Engl. J. Med. 360, 753-764 (2009).

10 Lenzini P, Wadelius M, Kimmel S et al. Integration of genetic, clinical, and laboratory data to refine warfarin dosing. Clin. Pharmacol. Ther. 87, 572-578 (2010).

11 Bress A, Patel SR, Perera MA, Campbell RT, Kittles RA, Cavallari LH. Effect of NQO1 and CYP4F2 genotypes on warfarin dose requirements in Hispanic-Americans and African-Americans. Pharmacogenomics 13(16), 1925-1935 (2012).

12 Duconge J, Cadilla CL, Seip RL, Ruaño G. Why admixture matters in genetically-guided therapy: missed targets in 
the COAG and EU-PACT trials. PR Health Sci. J. 34(3), 175-177 (2015).

13 Villagra D, Duconge J, Windemuth A et al. CYP2C9 and VKORC1 genotypes in Puerto Ricans: a case for admixturematching in clinical pharmacogenetic studies. Clin. Chim. Acta 411(17), 1306-1311 (2010).

14 Duconge J, Ruaño G. Emerging role of admixture in the pharmacogenetics of Puerto Rican Hispanics. J. Pharmacogenom. Pharmacoproteomics 1(101), pii:1000101 (2010).

15 Duconge J. Population heterogeneity and genomic admixture: relevance for global pharmacogenetics. J. Pharmacogenom. Pharmacoproteomics 5 , 5 (2014).

http://dx.doi.org/10.4172/2153-0645.1000e141

16 Suarez-Kurtz G. Pharmacogenomics in Admixed Populations. Landes Bioscience, TX, USA (2007).

17 Suarez-Kurtz G, Pena SD. Pharmacogenomics in the Americas: the impact of genetic admixture. Curr. Drug Targets 7, 1649-1658 (2006).

18 Claudio-Campos K, Duconge J, Cadilla CL, Ruaño G. Pharmacogenetics of drug metabolizing enzymes in U.S Hispanics. Drug Metabol. Personal. Ther. 30 (2), 87-105 (2015). 